\title{
Down syndrome critical region 1 positively correlates with angiogenesis in hypopharyngeal cancer
}

\author{
CHAO LV ${ }^{1}$, DAYU LIU ${ }^{2}$ and XIAONA WEI ${ }^{1}$ \\ ${ }^{1}$ Department of Otorhinolaryngology, Yantaishan Hospital, Yantai, Shandong 264000; ${ }^{2}$ Department of Otorhinolaryngology, \\ Qilu Hospital, Shandong University, Jinan, Shandong 250012, P.R. China
}

Received January 31, 2015; Accepted December 29, 2015

DOI: $10.3892 / \mathrm{mmr} .2016 .5989$

\begin{abstract}
Hypopharyngeal carcinoma has one of the highest mortality rates of head and neck cancer, therefore, the identification of markers associated with the pathogenesis and development of hypopharyngeal cancer is critical. Down syndrome critical region 1 (DSCR1) is associated with carcinogenesis and tumor growth in several types of malignancy. Activation of the vascular endothelial growth factor (VEGF) signaling pathway upregulates DSCR1. The aims of the present study were to determine the expression levels of DSCR1 and VEGF-C in hypopharyngeal cancer, and investigate the association between DSCR1 and angiogenesis in the disease. Tissue samples from 94 cases of pathologically confirmed hypopharyngeal squamous cell carcinoma were collected. The mRNA levels of DSCR1 and VEGF-C in cancerous and paracancerous tissues were examined using semi-quantitative reverse transcription-polymerase chain reaction. Microvessel density (MVD) was counted, according to the number of cluster of differentiation 34-positive cells. Spearman's correlation analysis was utilized to analyze the association between DSCR1 and angiogenesis. The relative mRNA expression levels of DSCR1 and VEGF-C, and the MVD were significantly increased in the cancerous tissue samples from the patients with hypopharyngeal cancer, compared with the paracancerous tissue samples from these patients. Higher levels of DSCR1 and increased MVD were associated with poorly differentiated tumors and lymph node metastasis. The mRNA expression levels of DSCR1 were positively correlated with the mRNA levels of VEGF-C in the cancerous tissues. The protein expression levels of DSCR1 were also positively correlated with MVD in the cancerous tissues. The results indicated that DSCR1 is involved in tumor angiogenesis in
\end{abstract}

Correspondence to: Dr Dayu Liu, Department of Otorhinolaryngology, Qilu Hospital, Shandong University, 107 Wenhua West Road, Jinan, Shandong 250012, P.R. China E-mail: entldy@aliyun.com

Key words: down syndrome critical region 1, vascular endothelial growth factor, angiogenesis, hypopharyngeal cancer patients with hypopharyngeal cancer, and is closely associated with the progression of the disease.

\section{Introduction}

Hypopharyngeal carcinoma has one of the lowest 5-year-survival rates of head and neck tumors (1). Early diagnosis of hypopharyngeal cancer is difficult, therefore the majority of these tumors are at an advanced stage when they are diagnosed. The 5-year-survival rates for patients treated with radiotherapy is $10-20 \%$, with surgery is $30-40 \%$, and with a combination of surgery and radiotherapy is $40-60 \%$ (2). Despite improved therapeutic strategies in previous years, there remains no effective treatment for this type of tumor. Thus, it is important to identify markers associated with the pathogenesis and development of hypopharyngeal carcinoma to enable earlier diagnosis and treatment.

Angiogenesis, the process whereby new capillaries form from pre-existing blood vessels, is key in physiological and pathological conditions. Angiogenesis is important in normal development and wound healing, and is critical for tumor growth, invasion and metastasis (3-5). Vascular endothelial growth factor (VEGF) stimulates tumor angiogenesis (6). Three types of VEGF receptor (VEGFR) tyrosine kinases have been identified, including VEGFR-1, VEGFR-2 (also termed kinase insert domain receptor) and VEGFR-3 $(7,8)$. The VEGF gene encodes five polypeptide growth factors, VEGF-A, -B, -C, -D and -E. VEGF-C, also termed VEGF-related protein, has been characterized as a lymphangiogenic and angiogenic growth factor (9). The binding of VEGF-C to VEGFR results in phosphorylation of the receptor. Activation of tyrosine kinases stimulates endothelial cell mitosis, promotes tumor vascular endothelial cell proliferation and induces tumor angiogenesis. VEGF-C and the VEGFR are expressed in lymphatic endothelial cells and a variety of tumor cells (10). Activation of the VEGF-C/VEGFR signaling pathway stimulates the formation of blood vessels and enhances cancer cell migratory and invasive abilities. Thus, activation of this signaling pathway facilitates cancer cell metastasis (8).

Down syndrome critical region 1 (DSCR1), induced during cell adaptation to oxidative stress, belongs to a family of evolutionarily conserved small proteins. The DSCR1 gene, located on human chromosome 21 , encodes a protein, which binds directly to the calcineurin catalytic A subunit (11). 
DSCR1 also has a regulatory role in calcineurin-mediated signaling $(11,12)$. It is expressed at high levels in the brain, heart and skeletal muscle $(11,13)$. DSCR1 has been implicated in several diseases, including Alzheimer's disease, Down syndrome, cardiac hypertrophy, gastrointestinal abnormalities and immune system deficiencies (11,14-16). In addition to its involvement in endothelial cell migration and angiogenesis $(17,18)$, DSCR1 has also been associated with carcinogenesis and tumor growth. Epidemiological studies have demonstrated that individuals with Down's syndrome are at higher risk of leukemia and testicular cancer, compared with the general population (19-21). However, certain studies have suggested that DSCR1 exerts a tumor suppressive effect in cancer via inhibiting tumor growth $(22,23)$. Therefore, the role of Down's syndrome and DSCR1 in cancer development remains controversial.

Our previous study demonstrated that the protein expression levels of DSCR1 were significantly higher in cancerous tissues, compared with paracancerous tissues in patients with hypopharyngeal cancer (24). DSCR1 was expressed in $94.9 \%$ of the cancerous tissues and in $35.9 \%$ of the paracancerous tissues examined. The elevated protein levels of DSCR1 were positively correlated with poorly differentiated tumors and advanced tumor-lymph node-metastasis (TNM) stage in these patients. Whether DSCR1 is also upregulated at the transcriptional level in hypopharyngeal cancer remains to be elucidated. Studies have demonstrated that the DSCR1 gene is a downstream target of VEGF signaling, whereby activation upregulates the expression of DSCR1 $(18,25,26)$. However, whether the mRNA levels of DSCR1 positively correlate with the mRNA levels of VEGF-C in hypopharyngeal cancer remains to be elucidated. The present study hypothesized that the mRNA levels of DSCR1 in hypopharyngeal cancerous tissues are increased, and that this elevation in the expression of DSCR1 is associated with increased expression levels of VEGF-C. Therefore, the aims of the present study were to determine the expression levels of DSCR1 and VEGF-C in hypopharyngeal cancer, and to investigate the association between DSCR1 and angiogenesis in this disease.

\section{Patients and methods}

Patients. The present study was approved by the Institutional Ethics Committee of Qilu Hospital, Shandong University, (Jinan, China), and complied with the 1964 Helsinki declaration (27) and its later amendments or comparable ethical standards. Written informed consent was obtained by the recruited patients or their family members. Between February 2009 and October 2010, 94 cases of pathologically confirmed hypopharyngeal squamous cell carcinoma were examined in the Department of Otolaryngology and Head and Neck Surgery, Qilu Hospital, Shandong University (Jinan, China). The inclusion criteria were as follows: Age $>18$ years; tumor type, primary hypopharynx carcinoma; patients underwent surgical treatment; pathological diagnosis of squamous cell carcinoma; patients underwent radiotherapy and chemotherapy. The patients were divided into two groups, according to age ( $<60$ or $\geq 60$ years old). Histological differentiation of the cancer was defined as well-differentiated, moderately differentiated or poorly differentiated. TNM staging was performed, according to the 1997 criteria of the Union for International Cancer Control (28). The tumors were also classified by their mode of growth, as either exogenous or infiltrative. In addition, patient smoking histories were recorded.

Tissue specimen collection. All tissue specimens were collected from the patients with primary hypopharyngeal tumors during surgery. The cancerous tissue was collected from the center of the hypopharyngeal tumor. The tissue adjacent to the tumor (paracancerous tissue) was collected, from a site 10-20 $\mathrm{mm}$ from the margin of the tumor. Following removal of the tissue, the specimens were immediately rinsed in RNA enzyme-free saline (Shanghai Sangon Biotechnology Co., Shanghai, China), treated with $0.1 \%$ diethylpyrocarbonate, and the impurities, including blood clots and eschar, were carefully removed. The specimens were immediately transferred to RNA enzyme-free Eppendorf tubes and preserved in liquid nitrogen. The remaining tumor tissue was prepared for histological or immunohistochemical examination.

Semi-quantitative reverse transcription-polymerase chain reaction $(R T-P C R)$. A total of 65 frozen tissue samples were prepared. A total of $100 \mathrm{mg}$ of each tissue specimen was homogenized in a tissue homogenizer containing $600 \mu \mathrm{l}$ guanidium thiocyanate denaturing solution. The organic and aqueous phases were separated by centrifugation for $5 \mathrm{~min}$ at $13,350 \mathrm{x}$. The RNA-containing aqueous phase was carefully transferred into a fresh centrifuge tube, and the RNA was precipitated by addition of an equal volume of cold isopropanol. Subsequently, the samples were centrifuged ar $13,350 \mathrm{x} \mathrm{g}, 4^{\circ} \mathrm{C}$ for $20 \mathrm{~min}$ to pellet RNA. Following washing, RNA was dissolved in RNase free water and stored at $-20^{\circ} \mathrm{C}$. Total RNA from the cancerous and paracancerous tissues was extracted using a TRIzol total RNA extraction kit (Shanghai Shenggong Biology Engineering Technology Service, Ltd., Shanghai, China). First strand complementary DNA (cDNA) was reverse transcribed using a PrimeScript $^{\mathrm{TM}}$ High Fidelity RT-PCR kit (Takara Biotechnology Co., Ltd., Dalian, China). The cDNA of the target genes was amplified using PCR master mix purchased from Takara Bio, Inc. (Otsu, Japan). The target gene primers $(1 \mu \mathrm{l})$ were purchased from Shanghai Sangon Biotechnology Co. Reverse transcription reactions were performed at $30^{\circ} \mathrm{C}$ for $15 \mathrm{~min}, 56^{\circ} \mathrm{C}$ for $35 \mathrm{~min}$, $99^{\circ} \mathrm{C}$ for $5 \mathrm{~min}$ and $5^{\circ} \mathrm{C}$ for $5 \mathrm{~min}$. The conditions used for the PCR were as follows: $94^{\circ} \mathrm{C}$ for $2 \mathrm{~min} ; 94^{\circ} \mathrm{C}$ for $45 \mathrm{sec}, 55^{\circ} \mathrm{C}$ for $45 \mathrm{sec}, 72^{\circ} \mathrm{C}$ for $90 \mathrm{sec}$ for 30 cycles and $72^{\circ} \mathrm{C}$ for $5 \mathrm{~min}$. The sequences of the primers were as follows: Sense 5'-TGCGAC CCCAGTCATAAACTA-3' and antisense 5'-CCATTTCCT CTTCTTCCT-3' for DSCR1; sense 5'-CTCAAAAGTTATT TTAATAACAGG-3' and antisense 5'-GTTAGGACTTATTCC TGTCATTA-3' for VEGF-C; and sense 5'-ATCATGTTTGAG ACCTTCAACA-3' and antisense 5'-CATCTCTTGCTCGAA GTCCA-3' for $\beta$-actin. The mRNA expression of $\beta$-actin served as a housekeeping control. Following amplification, the PCR products were resolved by running them on a $2 \%$ agarose gel (Shanghai Sangon Biotechnology Co.), and the relative mRNA expression was calculated using an electrophoresis gel quantitative image analyzer (Kodak, Rochester, NY, USA).

Immunohistochemistry. Immunohistochemistry was performed using a PV9000 staining kit (Beijing Zhongpin 
Jinqiao, Biotechnology Co., Ltd., Beijing, China), according to the manufacturer's protocols. Paraffin sections were placed in xylene solution for 10-15 min twice at room temperature. Subsequently, sections were dehydrated in a gradient series of 70-100\% ethanol. Following deparaffinization and hydration, tissue sections were incubated for $10 \mathrm{~min}$ in $3 \%$ hydrogen peroxide in methanol and were washed three times with phosphate-buffered saline (PBS). Subsequent to antigen retrieval, the slides were again washed three times for 5 min with PBS. The slides were then blocked with blocking solution A (normal goat serum; Beijing Zhongshan Jinqiao Biological Technology Co., Ltd., Beijing, China) for $15 \mathrm{~min}$ at room temperature, and incubated with specific primary antibodies overnight at $4^{\circ} \mathrm{C}$. The antibodies used were as follows: Rat monoclonal anti-human cluster of differentiation (CD)34 (QBEnd/10; 1:250; cat. no. ZM-0046; Beijing Zhongshan Jinqiao Biotechnology Co., Ltd.) and polyclonal rabbit anti-human DSCR1 (DCT3; 1:250; provided by Prof Philip Brandt, Columbia University, New York, NY, USA). The slides were then incubated with solution B for $20 \mathrm{~min}$ at room temperature. Following washing with PBS three times for $5 \mathrm{~min}$, the slides were incubated with solution $\mathrm{C}$ for $20 \mathrm{~min}$ at room temperature. The slides were stained with Dolichos biflorus agglutinin (Shanghai Chemical Reagent Co., Ltd., Shanghai, China) and hematoxylin and eosin and dehydrated prior to observation under a light microscope (Olympus CX31; Olympus Corporation, Tokyo, Japan). PBS served as the blank control, normal goat serum as the negative control and human brain tissue as the positive control. Brain tissue was obtained from the pathological tissue library of Shandong University (Jinan, China).

Determination of microvessel density (MVD). In the CD34 antibody-stained sections, the presence of brown granules in the cytoplasm indicated positively stained vascular endothelial cells. Positive cells with a clear background and a distinct boundary or basement membrane, including single-stained endothelial cells, were classed as one vessel unit, regardless of the formation of the vascular lumen or whether red blood cells were present within the lumen. For vessels containing lumens over eight red blood cells in size, the muscular layer was not counted. The tumor region and the interstitial region in all tissue sections were examined, with the exception of the surrounding areas, disrupted tissue or areas of tumor necrosis. LEICA Qwin 3.31 automatic image software (Leica Microsystems GmbH, Wetzlar, Germany) was used to count the MVD. The MVD was counted in five randomly-selected separate fields in each section using a light microscope (Olympus CX31; Olympus Corporation) at magnification, $\mathrm{x} 400$. The mean value of the five counts was determined to be the MVD.

Calculations of DSCR1-positive cells. In the DSCR1 antibody-stained sections, the presence of brown granules in the cytoplasm of the cells was considered to be positive for DSCR1. Tumor cells in each field (500) were examined from a total of five randomly-selected separate fields in each section at magnification, $x 400$. The positive cells were counted by two pathologists using a double-blind method, and the mean values were calculated. Sections containing $<10 \%$ positively stained cells were defined as negative (-), those with $10-20 \%$ positively
Table I. Characteristics of patients with hypopharyngeal cancer.

\begin{tabular}{|c|c|c|}
\hline Variable & Number & $\%$ \\
\hline Total & 94 & 100 \\
\hline \multicolumn{3}{|l|}{ Age (years) } \\
\hline$<60$ & 49 & 52.13 \\
\hline$\geq 60$ & 45 & 47.87 \\
\hline \multicolumn{3}{|l|}{ Gender } \\
\hline Male & 89 & 94.68 \\
\hline Female & 5 & 5.34 \\
\hline \multicolumn{3}{|l|}{ Histological differentiation } \\
\hline Well-differentiated & 30 & 31.91 \\
\hline Moderately differentiated & 43 & 45.74 \\
\hline Poorly differentiated & 21 & 22.34 \\
\hline \multicolumn{3}{|l|}{ TNM stage } \\
\hline I-II & 8 & 8.51 \\
\hline III-IV & 86 & 91.49 \\
\hline \multicolumn{3}{|l|}{ Lymph node metastasis } \\
\hline Yes & 54 & 57.45 \\
\hline No & 40 & 42.55 \\
\hline \multicolumn{3}{|l|}{ Growth mode } \\
\hline Exogenous & 49 & 52.13 \\
\hline Infiltrative & 45 & 47.87 \\
\hline \multicolumn{3}{|l|}{ Location } \\
\hline Piriform fossa & 75 & 79.79 \\
\hline Posterior pharyngeal wall & 15 & 15.96 \\
\hline Postcricoid region & 4 & 4.26 \\
\hline \multicolumn{3}{|l|}{ Smoking } \\
\hline Yes & 69 & 73.40 \\
\hline No & 25 & 26.60 \\
\hline
\end{tabular}

TNM, tumor-node-metastasis.

stained cells were weakly positive $( \pm)$, those with $20-40 \%$ positively stained cells were positive $(+)$, those with $40-60 \%$ positively stained cells were medium positive $(++)$ and those with $>60 \%$ positively stained cells were considered strongly positive $(+++)$.

Statistical analysis. Data were analyzed using SAS, version 8.2 statistical software (SAS Institute, Inc., Cary, NC, USA). All continuous data are expressed as the mean \pm standard deviation. Student's t-test was used for the comparison between two groups. Spearman's correlation analysis was utilized to analyze the association between DSCR1 and angiogenesis. $\mathrm{P}<0.05$ was considered to indicate a statistically significant difference.

\section{Results}

Patient characteristics. A total of 94 cases of hypopharyngeal squamous cell carcinoma were examined in the present study. The mean age of the patients was 47 years, $52.13 \%$ were $<60$ years, $47.87 \%$ were $\geq 60$ years; $94.68 \%$ were males and 
A

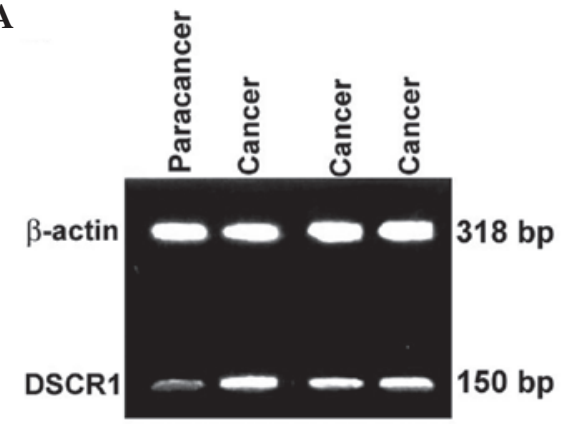

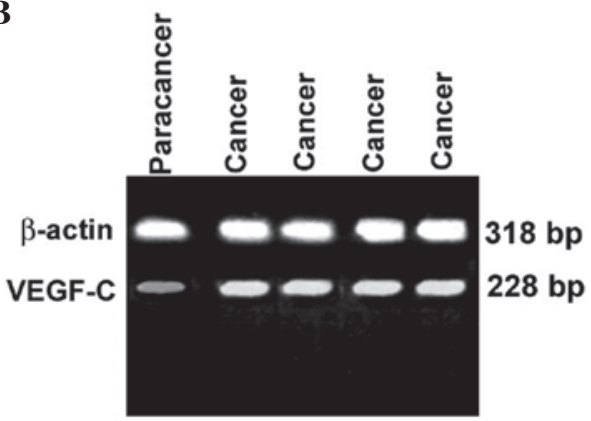

C

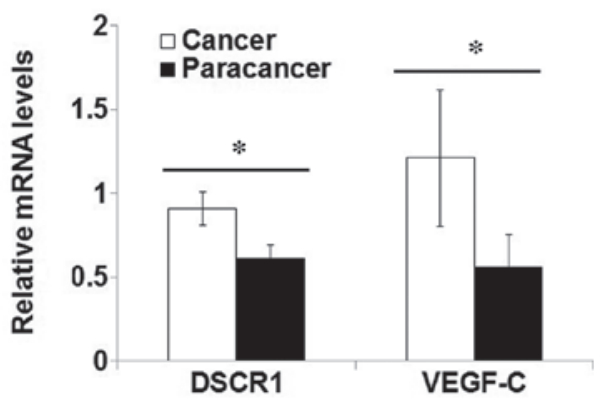

Figure 1. Representative images of the mRNA expression levels of DSCR1 and VEGF-C in patients with hypopharyngeal cancer. Semi-quantitative reverse transcription-PCR was performed, and following amplification, the PCR products of (A) DSCR1 and (B) VEGF-C were resolved on a $2 \%$ agarose gel. (C) Relative mRNA expression levels were calculated using an electrophoresis gel quantitative image analyzer. mRNA expression of $\beta$-actin was used as a housekeeping control. Data are expressed as the mean \pm standard deviation ("P<0.05). DSCR1, Down syndrome critical region 1; VEGF, vascular endothelial growth factor; PCR, polymerase chain reaction.

$5.34 \%$ were females (Table I). Based on histological differentiation, there were 30 well-differentiated cases $(31.91 \%)$, 43 moderately differentiated cases $(45.74 \%)$ and 21 poorly differentiated cases $(22.34 \%)$. A total of eight cases $(8.51 \%)$ were stages I-II and 86 (91.49\%) were stages III-IV. Lymph node metastasis was observed in 54 cases $(57.45 \%)$. Exogenous tumor growth was observed in 49 cases, whereas infiltrative growth was observed in 45 cases. The majority of the tumors were located at the piriform fossa $(79.79 \%)$, and the remaining were localized to the posterior pharyngeal wall $(15.96 \%)$ or the postcricoid region (4.26\%). Of the patients included in the present study 69 (73.4\%) had a history of smoking (Table I).

mRNA expression levels of DSCR1 and VEGF-C, and MVD are increased in cancerous tissues, compared with paracancerous tissue in hypopharyngeal cancer. Cancerous tissue was collected from 43 patients and paracancerous tissue was collected from 21 of these patients. The tissue adjacent to the cancer was pathologically confirmed as normal pharyngeal mucosa. In patients with hypopharyngeal squamous cell carcinoma, the overall mean relative mRNA expression levels of DSCR1 in the cancerous tissue were significantly higher than in the paracancerous tissue ( $0.91 \pm 0.10$ vs. $0.61 \pm 0.08$, respectively; $\mathrm{P}=0.011$; Fig. 1). In addition, the overall mean relative mRNA expression levels of VEGF-C in the cancerous tissue was significantly higher than in the paracancerous tissue $(1.21 \pm 0.41$ vs. $0.56 \pm 0.19$, respectively; $\mathrm{P}=0.011$; Fig. 1 ). The MVD in the 78 cancerous and paracancerous tissue samples from the patients with hypopharyngeal squamous cell carcinoma patients were also examined. The MVD was significantly higher in the cancerous tissues, compared with the paracancerous tissue $(17.61 \pm 10.59$ vs. 12.59 \pm 7.77 , respectively; $\mathrm{P}=0.011$; Fig. 2).
Expression levels of DSCR1 correlate with the progression of hypopharyngeal cancer. To define the role of DSCR1 in the progression of cancer of the laryngopharynx, the expression profiles of DSCR1 (Fig. 2) were analyzed, according to tumor differentiation and TNM stage. The results indicated that higher mRNA expression levels of DSCR1 were associated with poor tumor differentiation $(\mathrm{P}=0.033)$ and lymph node metastasis $(\mathrm{P}=0.042$; Table II). No statistically significant associations were found between the protein levels of DSCR1 and the different modes of tumor growth or patient smoking history (Table II).

MVD is correlated with the progression of hypopharyngeal cancer. To determine the association between tumor angiogenesis or lymphangiogenesis with the clinical characteristics of patients with hypopharyngeal squamous cell carcinoma, the MVDs in tumor tissues from 94 patients were examined (Fig. 2). Compared with patients with a lower intratumoral MVD, the tumor tissues in patients with a higher intratumoral MVD were poorly differentiated with a higher TNM stage and increased lymph node metastasis (all $\mathrm{P}<0.05$; Table III). The MVDs were similar in patients with different tumor growth modes and in smokers, compared with non-smokers.

DSCR1 is positively correlated with tumor angiogenesis in hypopharyngeal cancer. The mRNA expression levels of DSCR1 were positively correlated with the mRNA levels of VEGF-C in the cancerous tissue of patients with hypopharyngeal squamous cell carcinoma, $(\mathrm{r}=0.578 ; \mathrm{P}<0.001)$. As shown in Fig. 3, the MVDs in the cancerous tissue were 10.7 \pm 4.44

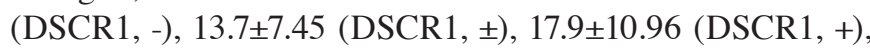
26.5 \pm 10.55 (DSCR1, ++) and 29.1 \pm 11.65 (DSCR1, +++). The 
Table II. mRNA and protein levels of DSCR1 in patients with hypopharyngeal cancer.

\begin{tabular}{|c|c|c|c|c|}
\hline \multirow[b]{2}{*}{ Variable } & \multicolumn{2}{|c|}{ DSCR1 mRNA } & \multirow[b]{2}{*}{$\mathrm{n}$} & \multirow[b]{2}{*}{$\%$} \\
\hline & Cancer tissue $(\mathrm{n}=43)$ & P-value & & \\
\hline Total & $0.91 \pm 0.10$ & & 35 & 81.40 \\
\hline Histological differentiation & & $0.033^{\mathrm{a}}$ & & \\
\hline Well-differentiated & $0.73 \pm 0.11$ & & 8 & 18.60 \\
\hline Moderately-differentiated & $0.79 \pm 0.09$ & & 19 & 44.19 \\
\hline Poorly-differentiated & $0.93 \pm 0.15$ & & 7 & 16.28 \\
\hline TNM stage & & 0.121 & & \\
\hline I-II & $0.89 \pm 0.13$ & & 4 & 9.30 \\
\hline III-IV & $0.92 \pm 0.08$ & & 31 & 72.09 \\
\hline Lymph node metastasis & & $0.042^{\mathrm{a}}$ & & \\
\hline Yes & $0.93 \pm 0.11$ & & 26 & 60.47 \\
\hline No & $0.79 \pm 0.07$ & & 9 & 20.93 \\
\hline Growth mode & & 0.138 & - & - \\
\hline Exogenous & $0.90 \pm 0.07$ & & - & - \\
\hline Infiltrative & $0.89 \pm 0.17$ & & - & - \\
\hline
\end{tabular}

mRNA levels are expressed as the mean \pm standard deviation. DSCR1, Down syndrome critical region 1; TNM, tumor-node-metastasis. ${ }^{\mathrm{a}} \mathrm{P}<0.05$.
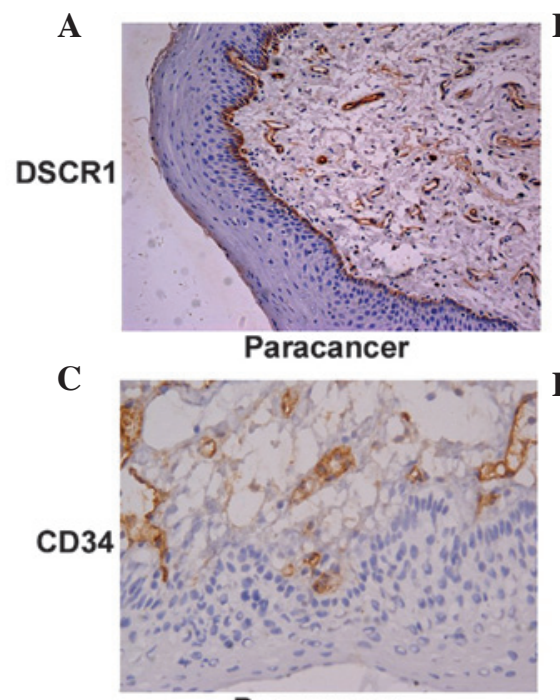

Paracancer
B

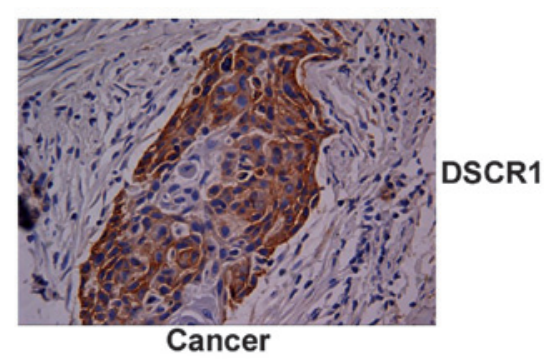

D

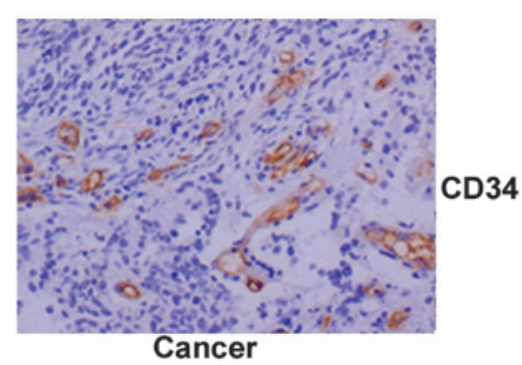

Figure 2. Representative images of the protein expression levels of DSCR1 and microvessel density in patients with hypopharyngeal cancer. Proteins were examined in cancerous and paracancerous tissues. Immunohistochemistry was performed to determine the levels of (A and B) DSCR1 and (C and D) CD34. Magnification, x400. DSCR1, Down syndrome critical region 1; CD34, cluster of differentiation 34.

MVDs increased significantly with increasing protein levels $(\mathrm{P}<0.05)$. A significant correlation was found between the protein levels of DSCR1 and MVD in the patients with cancer $(r=0.689 ; \mathrm{P}<0.001)$. These results indicated that higher protein expression levels of DSCR1 were significantly associated with angiogenesis in laryngopharynx cancer.

\section{Discussion}

In the present study, the expression levels of DSCR1 in patients with hypopharyngeal squamous cell carcinoma were examined.
The results demonstrated that the relative mRNA expression levels of DSCR1 in the cancerous tissues were significantly higher than those in the paracancerous tissues. This finding is consistent with a previous study, which demonstrated that the protein levels of DSCR1 were significantly increased in hypopharyngeal cancer (24). This previous study also indicated that elevated protein expression levels of DSCR1 was correlated with poor tumor differentiation and advanced TNM stage (28). In the present study, elevated mRNA expression levels of DSCR1 were also associated with poor tumor differentiation and advanced disease stage, which suggested that the upregulation of DSCR1 
Table III. MVD in patients with hypopharyngeal cancer.

\begin{tabular}{|c|c|c|c|}
\hline Variable & Number & MVD & P-value \\
\hline Total & 94 & $17.57 \pm 10.61$ & \\
\hline Age (years) & & & 0.639 \\
\hline$<60$ & 49 & $17.99 \pm 10.49$ & \\
\hline$\geq 60$ & 45 & $17.23 \pm 11.34$ & \\
\hline Gender & 94 & & 0.588 \\
\hline Male & 89 & $17.64 \pm 10.53$ & \\
\hline Female & 5 & $18.88 \pm 9.89$ & \\
\hline Histological differentiation & & & 0.037 \\
\hline Well-differentiated & 30 & $20.48 \pm 13.61$ & \\
\hline Moderately differentiated & 43 & $18.58 \pm 10.35$ & \\
\hline Poorly differentiated & 21 & $16.29 \pm 9.98$ & \\
\hline TNM stage & & & 0.045 \\
\hline I-II & 8 & $16.13 \pm 9.61$ & \\
\hline III-IV & 76 & $18.43 \pm 11.61$ & \\
\hline Lymph node metastasis & & & 0.031 \\
\hline Yes & 54 & $18.96 \pm 11.63$ & \\
\hline No & 40 & $16.17 \pm 9.61$ & \\
\hline Location & & & 0.386 \\
\hline Piriform fossa & 75 & $17.20 \pm 10.23$ & \\
\hline Posterior pharyngeal wall & 15 & $18.10 \pm 11.13$ & \\
\hline Postcricoid region & 4 & $18.75 \pm 11.88$ & \\
\hline Smoking & & & 0.874 \\
\hline Yes & 69 & $17.79 \pm 10.21$ & \\
\hline No & 25 & $17.11 \pm 10.79$ & \\
\hline
\end{tabular}

MDV values are expressed as the mean \pm standard deviation. MVD, microvessel density; TNM, tumor-node-metastasis.

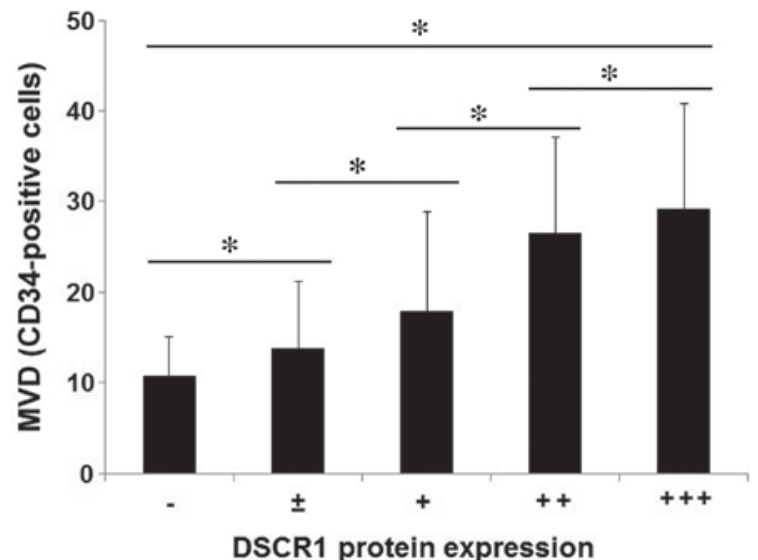

Figure 3. MVD, determined by the number of CD34-positive cells, in cancer tissue is positively correlated with protein levels of DSCR1. Data are expressed as the mean \pm standard deviation ( $\mathrm{P}<0.05)$. -, negative; \pm , weakly positive; +, positive; ++, medium positive; +++, strongly positive; MVD, microvessel density; CD34, cluster of differentiation 34; DSCR1, Down syndrome critical region 1 .

is important in the pathogenesis and progression of hypopharyngeal cancer. DSCR1 may serve as a useful marker in the early diagnosis and monitoring of this type of cancer.
A previous study examining cancer incidence in individuals with Down's syndrome indicated that the overall risk of cancer was similar between the these individuals and the general population. However, the individuals with Down's syndrome had a significantly higher risk of leukemia, with a standardized incidence ratio (SIR) of $10.5,95 \%$ confidence interval (CI) of 6.6-15.8, and testicular cancer (SIR, 4.8; 95\% CI, 1.8-10.4) (21). Another study demonstrated that the targeted deletion of DSCR1 in mice inhibited the formation of new tumor vasculature and suppressed tumorigenesis. Treatment with the calcineurin-specific inhibitor, cyclosporin A attenuated this endothelial defect and promoted tumor growth (29). However, Baek et al (22) reported that the incidence of cancer was significantly reduced in individuals with Down's syndrome, and that the upregulation of DSCR1 inhibited tumor growth. Sussan et al (23) also suggested that trisomy reduced the numbers of intestinal tumors in a mouse model of Down's syndrome. These reports indicate that the role of DSCR1 in cancer is controversial, and further investigations are required to elucidate the function of DSCR1 in carcinogenesis.

It is well-known that the expression of VEGF-C is increased in various types of human malignancy $(30,31)$. The levels of VEGF-C in tumor tissues are significantly correlated with regional angiogenesis $(30,31)$. Bunone et al (30) demonstrated 
that the mRNA and protein expression levels of VEGF-C were upregulated in thyroid carcinoma, and Yonemura et al (31) reported that the mRNA and protein expression levels of VEGF-C were also increased in gastric carcinoma. Similarly, the present study demonstrated that, in patients with hypopharyngeal cancer, the relative mRNA expression levels of VEGF-C were significantly higher than in the normal tissues suggesting that the levels of VEGF-C are elevated in hypopharyngeal cancer, concordant with previous reports in other types of cancer $(30,31)$.

In the present study, a statistically significant positive correlation was observed between the mRNA expression levels of DSCR1 and VEGF-C in the cancerous tissue from patients with hypopharyngeal cancer. The mRNA expression levels of DSCR1 were significantly higher in tissues expressing upregulated levels of VEGF-C. In addition, the MVD in the laryngopharynx cancerous tissue was significantly increased, compared with that in the paracancerous tissue $(\mathrm{P}<0.05)$, and the protein expression of DSCR1 in the cancerous tissue were significantly correlated with MVD ( $\mathrm{r}=0.689 ; \mathrm{P}<0.001)$. Yao et al (18) demonstrated that DSCR1 is upregulated in endothelial cells upon stimulation with VEGF, TNF- $\alpha$ and A23187 treatment. The upregulation of DSCR1 is suppressed by inhibitors of the calcineurin-nuclear factor of activated $\mathrm{T}$ cell (NFAT) signaling pathway, inhibition of protein kinase $\mathrm{C}$ (PKC) and $\mathrm{Ca}^{2+}$ chelation (32). Furthermore, Hesser et al (13) conducted a genome-wide analysis of genes, which are regulated by VEGF in endothelial cells. This analysis identified DSCR 1 as one of the most frequently induced targets of VEGF. The results of our previous and present studies are consistent with the report by Yao et al (18). These findings indicated that DSCR1 is involved in tumor angiogenesis, and suggested that DSCR1 is important in angiogenesis, specifically in hypopharyngeal carcinoma, and may be a promising therapeutic target.

In response to various stimuli, tumor cells secrete a large quantity of VEGF via either paracrine or autocrine mechanisms. VEGF binds to VEGFR-1 and VEGFR-2, leading to the activation of downstream signaling, including phosphatidylinositol 3-kinase/protein kinase B, phospholipase C- $\gamma, \mathrm{PKC}$, mitogen activated protein kinases and calcineurin (33-36). Abe and Sato (26) reported that the DSCR1 gene is an important downstream target of VEGF in human endothelial cells. VEGF enhances calcineurin activity (18). Calcineurin is involved in the regulation of a variety of cellular activities, including cell maturation, survival, proliferation and functional activities (18). Calcineurin binds to and activates NFAT, which subsequently translocates to the nucleus. NFAT promotes the transcription of various target genes by binding to the promoter and enhancer regions of these genes $(11,15,37)$. Previous studies have demonstrated that the binding of calcineurin to DSCR1 enhances the expression of DSCR1, and the upregulation of DSCR1 serves as a negative regulator of calcineurin activity, resulting in a negative feedback loop between DSCR1 and calcineurin A signaling (11,15,37). This may represent a potential molecular mechanism underlying the association between DSCR1 and angiogenesis.

It has been suggested that exposure of the active site during the binding of calcineurin to $\mathrm{Ca}^{2+} /$ calmodulin promotes calcineurin to bind with DSCR1. In addition to directly inhibiting calcineurin activity, mutational studies have demonstrated that
DSCR 1 competes with NFAT in binding to the same anchor points on the calcineurin protein. This is one of the molecular mechanisms by which DSCR1 inhibits the activity of the calcineurin/NFAT signaling pathway (38). DSCR1 also inhibits the phosphatase activity of calcineurin (36). As a downstream target of the calcineurin/NFAT signaling pathway, DSCR1 is activated by a series of factors associated with NFAT signaling, including VEGF, angiotensin-II, TNF- $\alpha$, thrombin and calcium ion carriers (39).

Although the present study demonstrated important findings, including the correlation between the mRNA and protein levels of DSCR1, angiogenesis and the progression of hypopharyngeal squamous cell carcinoma progression, the study had certain limitations. For example, the sample size was relatively small and patient follow-up information was absent; therefore, evaluation of correlations between patient survival times and the expression levels of DSCR1 or VEGF-C were not performed. In addition, the present study did not investigate in detailed the molecular mechanism(s) by which DSCR1 regulates angiogenesis in hypopharyngeal cancer. Further investigations are ongoing to determine the molecular signaling pathways involved in DSCR1-induced tumor angiogenesis and lymphangiogenesis.

In conclusion, the present study revealed that the mRNA expression levels of DSCR1 and VEGF-C were upregulated in cancerous tissue samples from patients with hypopharynx cancer. These elevated expression levels were correlated with poorly differentiated tumors and advanced TNM stage, and the expression levels of DSCR1 were positively correlated with angiogenesis in the tumor tissues. DSCR1 is likely to be important during the progression of hypopharyngeal cancer by regulating tumor angiogenesis and lymphangiogenesis. Thus, DSCR1 may be a promising marker for early diagnosis and early management of hypopharyngeal cancer.

\section{Acknowledgements}

The authors would like to thank Medjaden Bioscience Limited for assisting in the preparation of this manuscript.

\section{References}

1. Newman JR, Connolly TM, Illing EA, Kilgore ML, Locher JL and Carroll WR: Survival trends in Hypopharyngeal cancer: A population-based review. Laryngoscope 125: 624-629, 2015.

2. Berrino F and Gatta G: Variation in survival of patients with head and neck cancer in Europe by the site of origin of the tumours. EUROCARE working group. Eur J Cancer 34: 2154-2161, 1998.

3. Li X, Liu X, Wang J, Wang Z, Jiang W, Reed E, Zhang Y, Liu Y and Li QQ: Thalidomide down-regulates the expression of VEGF and bFGF in cisplatin-resistant human lung carcinoma cells. Anticancer Res 23: 2481-2487, 2003.

4. Folkman J: Angiogenesis in cancer, vascular, rheumatoid and other disease. Nat Med 1: 27-31, 1995.

5. Carmeliet P and Jain RK: Angiogenesis in cancer and other diseases. Nature 407: 249-257, 2000.

6. Ferrara N: VEGF and the quest for tumour angiogenesis factors. Nat Rev Cancer 2: 795-803, 2002.

7. Ferrara N, Gerber HP and LeCouter J: The biology of VEGF and its receptors. Nat Med 9: 669-676, 2003.

8. Su JL, Yen CJ, Chen PS, Chuang SE, Hong CC, Kuo IH, Chen HY, Hung MC and Kuo ML: The role of the VEGF-C/VEGFR-3 axis in cancer progression. Br J Cancer 96: 541-545, 2007.

9. Plate K: From angiogenesis to lymphangiogenesis. Nat Med 7: $151-152,2001$. 
10. Olsson AK, Dimberg A, Kreuger J and Claesson-Welsh L: VEGF receptor signalling - in control of vascular function. Nat Rev Mol Cell Biol 7: 359-371, 2006.

11. Fuentes JJ, Genescà L, Kingsbury TJ, Cunningham KW, Pérez-Riba M, Estivill X and de la Luna S: DSCR1, overexpressed in Down syndrome, is an inhibitor of calcineurin-mediated signaling pathways. Hum Mol Genet 9: 1681-1690, 2000.

12. Harris CD, Ermak G and Davies KJ: Multiple roles of the DSCR1 (Adapt78 or RCAN1) gene and its protein product calcipressin 1 (or RCAN1) in disease. Cell Mol Life Sci 62: 2477-2486, 2005.

13. Hesser BA, Liang XH, Camenisch G, Yang S, Lewin DA Scheller R, Ferrara N and Gerber HP: Down syndrome critical region protein 1 (DSCR1), a novel VEGF target gene that regulates expression of inflammatory markers on activated endothelial cells. Blood 104: 149-158, 2004.

14. Aynaci FM, Orhan F, Celep F and Karagüzel A: Frequency of cardiovascular and gastrointestinal malformations, leukemia and hypothyroidism in children with Down syndrome in Trabzon, Turkey. Turk J Pediatr 40: 103-109, 1998.

15. Rothermel B, Vega RB, Yang J, Wu H, Bassel-Duby R and Williams RS: A protein encoded within the Down syndrome critical region is enriched in striated muscles and inhibits calcineurin signaling. J Biol Chem 275: 8719-8725, 2000.

16. Vega RB, Rothermel BA, Weinheimer CJ, Kovacs A, Naseem RH, Bassel-Duby R, Williams RS and Olson EN: Dual roles of modulatory calcineurin-interacting protein 1 in cardiac hypertrophy. Proc Natl Acad Sci USA 100: 669-674, 2003.

17. Iizuka M, Abe M, Shiiba K, Sasaki I and Sato Y: Down syndrome candidate region 1, a downstream target of VEGF, participates in endothelial cell migration and angiogenesis. J Vasc Res 41: 334-344, 2004

18. Yao YG and Duh EJ: VEGF selectively induces Down syndrome critical region 1 gene expression in endothelial cells: A mechanism for feedback regulation of angiogenesis? Biochem Biophys Res Commun 321: 648-656, 2004.

19. Suzuki K, Nishimi D, Yagishita T, Takanami M and Hiruta N: Testicular tumor in Down syndrome. Int J Urol 12: 925-927, 2005.

20. Satgé D, Sommelet D, Geneix A, Nishi M, Malet P and Vekemans M: A tumor profile in Down syndrome. Am J Med Genet 78: 207-216, 1998.

21. Patja K, Pukkala E, Sund R, Iivanainen M and Kaski M: Cancer incidence of persons with Down syndrome in Finland: A population-based study. Int J Cancer 118: 1769-1772, 2006.

22. Baek KH, Zaslavsky A, Lynch RC, Britt C, Okada Y, Siarey RJ, Lensch MW, Park IH, Yoon SS, Minami T, et al: Down's syndrome suppression of tumour growth and the role of the calcineurin inhibitor DSCR1. Nature 459: 1126-1130, 2009.

23. Sussan TE, Yang A, Li F, Ostrowski MC and Reeves RH: Trisomy represses Apc (Min)-mediated tumours in mouse models of Down's syndrome. Nature 451: 73-75, 2008.

24. Lu C, Pan X, Gao J and Li Z: Study of expression and significance of DSCR1 gene in laryngopharynx cancer and peri-cancerous tissues. Lin Chung Er Bi Yan Hou Tou Jing Wai Ke Za Zhi 25: 848-850, 2011 (In Chinese).
25. Liu D, Jia H, Holmes DI, Stannard A and Zachary I: Vascular endothelial growth factor-regulated gene expression in endothelial cells: KDR-mediated induction of Egr3 and the related nuclear receptors Nur77, Nurr1 and Nor1. Arterioscler Thromb Vasc Biol 23: 2002-2007, 2003.

26. Abe M and Sato Y: CDNA microarray analysis of the gene expression profile of VEGF-activated human umbilical vein endothelial cells. Angiogenesis 4: 289-298, 2001.

27. World Medical Association: World Medical Association Declaration of Helsinki: Ethical principles for medical research involving human subjects. JAMA 310: 2191-2194, 2013.

28. Sobin L and Wittekind C (eds): UICC TNM classification of malignant tumours (5th Edition). Wiley Liss, New York, NY, pp138, 1997.

29. Ryeom S, Baek KH, Rioth MJ, Lynch RC, Zaslavsky A, Birsner A, Yoon SS and McKeon F: Targeted deletion of the calcineurin inhibitor DSCR1 suppresses tumor growth. Cancer Cell 13: 420-431, 2008.

30. Bunone G, Vigneri P, Mariani L, Butó S, Collini P, Pilotti S, Pierotti MA and Bongarzone I: Expression of angiogenesis stimulators and inhibitors in human thyroid tumors and correlation with clinical pathological features. Am J Pathol 155: 1967-1976, 1999.

31. Yonemura Y, Fushida S, Bando E, Kinoshita K, Miwa K, Endo Y, Sugiyama K, Partanen T, Yamamoto H and Sasaki T: Lymphangiogenesis and the vascular endothelial growth factor receptor (VEGFR)-3 in gastric cancer. Eur J Cancer 37: 918-923, 2001.

32. Pan MG, Xiong Y, Chen F: NFAT gene family in inflammation and cancer. Curr Mol Med 13:543-554, 2013

33. Kroll $\mathrm{J}$ and Waltenberger $\mathrm{J}$ : The vascular endothelial growth factor receptor KDR activates multiple signal transduction pathways in porcine aortic endothelial cells. J Biol Chem 272: 32521-32527, 1997.

34. Rousseau S, Houle F, Landry J and Huot J: p38 MAP kinase activation by vascular endothelial growth factor mediates actin reorganization and cell migration in human endothelial cells. Oncogene 15: 2169-2177, 1997.

35. Gerber HP, McMurtrey A, Kowalski J, Yan M, Keyt BA, Dixit V and Ferrara N: Vascular endothelial growth factor regulates endothelial cell survival through the phosphatidylinositol 3'-kinase/Akt signal transduction pathway. Requirement for Flk-1/KDR activation. J Biol Chem 273: 30336-30343, 1998.

36. Takahashi T, Ueno $\mathrm{H}$ and Shibuya M: VEGF activates protein kinase C-dependent, but Ras-independent Raf-MEK-MAP kinase pathway for DNA synthesis in primary endothelial cells. Oncogene 18: 2221-2230, 1999.

37. Kingsbury TJ and Cunningham KW: A conserved family of calcineurin regulators. Genes Dev 14: 1595-1604, 2000.

38. Chan B, Greenan G, McKeon F and Ellenberger T: Identification of a peptide fragment of DSCR1 that competitively inhibits calcineurin activity in vitro and in vivo. Proc Natl Acad Sci USA 102: 13075-13080, 2005.

39. Pinchai N, Perfect BZ, Juvvadi PR, Fortwendel JR, Cramer RA Jr, Asfaw YG, Heitman J, Perfect JR and Steinbach WJ: Aspergillus fumigatus calcipressin $\mathrm{CbpA}$ is involved in hyphal growth and calcium homeostasis. Eukaryot Cell 8: 511-519, 2009. 